\title{
Entretien avec le Professeur Paul-Laurent Assoun L'anthropologie psychanalytique
}

\section{Entrevista con el Profesor Paul-Laurent Assoun La antropología psicoanalítica}

\author{
Interview with Professor PaulLaurent Assoun. \\ The Psychoanalytical Anthropology [French version]
}

\section{Bruno Carignano}

Correspondencia:

brunocarignano@yahoo.com
Filiaciones Institucionales:

Universidad Nacional de Rosario (UNR) (Argentina)

Universidad de Ciencias Empresariales y Sociales (UCES) (Argentina)

Université de Paris (Francia)

RÉSumÉ: Cet entretien a eu lieu en France, à l'Université Denis Diderot - Paris 7 (aujourd'hui Université de Paris), le 22 juin 2019. Sa transcription au français et sa traduction à l'espagnol ont été faites par Bruno Carignano. Elles ont cherché à maintenir le plus fidèlement possible le registre de la transmission orale. Lors de l'entretien, Paul-Laurent Assoun traite divers sujets qui se rapportent à la discipline universitaire qu'il a introduit sous le nom d'anthropologie psychanalytique : sa liaison particulière à la psychanalyse freudienne et lacanienne ; la recherche en psychanalyse et l'Université dans la contemporanéité française ; les spécificités de la clinique psychanalytique et les phénomènes sociaux ; les relations du sujet de l'inconscient au collectif et la condition contemporaine ; le style borderline et autres phénomènes cliniques de la contemporanéité ; l'approche du féminin par la psychanalyse et le rapport de celle-ci avec les idéologies dans la considération du mouvement féministe. L'entretien a été revu par le Pr. Paul-Laurent Assoun.

Mots Clés: Anthropologie psychanalytique - Contemporanéité - Université - Style borderline - Le féminin

\section{Cómo citar:}

Carignano, B. (2020). Entretien avec le professeur Paul-Laurent Assoun. L'anthropologie psychanalytique en Revista Psicoanálisis en la Universidad $\mathrm{N}^{\circ} 4$. Rosario, Argentina,

UNR Editora. Pág 15-34

ISSN: 2683-9938 (en línea)

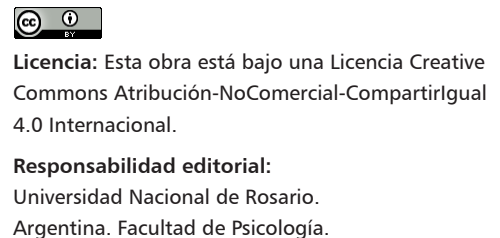

Commons Atribución-NoComercial-Compartirlgual

4.0 Internacional.

Universidad Nacional de Rosario.

Argentina. Facultad de Psicología.
Recibido:

$17-06-2020$

Aceptado:

$15-07-2020$

Publicado:

$05-10-2020$ 
Resumen: Esta entrevista tuvo lugar en Francia, en la Universidad Denis Diderot - París 7 (hoy Universidad de París) el 22 de junio de 2019. Su transcripción al francés y su traducción al español estuvieron a cargo de Bruno Carignano. En el transcurso de la entrevista, cuyo criterio fundamental ha sido mantener lo más fielmente posible el registro de la transmisión oral, Paul-Laurent Assoun aborda diversos temas relacionados con la disciplina universitaria que ha introducido bajo el nombre de antropología psicoanalítica: su modo peculiar de vinculación con el psicoanálisis freudiano y lacaniano, la investigación en psicoanálisis y la Universidad en la contemporaneidad francesa, las especificidades de la clínica psicoanalítica y los fenómenos sociales, las relaciones del sujeto del inconsciente con lo colectivo y la condición contemporánea, el estilo borderline y otros fenómenos clínicos de la contemporaneidad y, finalmente, el abordaje de lo femenino por parte del psicoanálisis y su relación con las ideologías en la consideración del movimiento feminista. La entrevista fue revisada por el Pr. Paul-Laurent Assoun.

Palabras claves: Antropología psicoanalítica - Contemporaneidad - Universidad Estilo borderline - Lo femenino

Aвstract: This interview took place in France, at Denis Diderot University - Paris 7 (nowadays Université de Paris), on June 22nd, 2019. Its transcription in French and its translation into Spanish were made by Bruno Carignano. The criterion was to maintain as much as possible of the oral transmission style. During the course of the interview, Paul-Laurent Assoun addresses various subjects related to the university discipline that he has introduced under the name of Psychoanalytical Anthropology: his particular connection to Freudian and Lacanian psychoanalysis; research in psychoanalysis and the University in contemporary France; the specificity of psychoanalytical clinic and social phenomena; the relations of the subject of the unconscious with the collective and the contemporary condition; the borderline style and other clinical phenomena of the contemporaneity. He also talks about the approach of the feminine by psychoanalysis and its relationship with ideologies in the consideration of the feminist movement. The interview was reviewed by Pr. Paul-Laurent Assoun.

KEYWORDS: Psychoanalytical Anthropology - Contemporaneity - University - Borderline style - The feminine 
Bruno Carignano : - Alors, mon idée, ce serait de vous poser quelques questions sur ces trois points suivants :

1. Le champ de l'anthropologie psychanalytique dans sa prise en compte des phénomènes cliniques et sociaux.

2. Le deuxième point est celui-ci : la femme, la féminité et l'CEdipe.

3. Et le troisième, ce serait : les états limites, structure psychopathologique et symptômes contemporains.

Tout d'abord, je souhaiterais vous demander comment vous est venue l'idée de commencer votre recherche dans ce domaine que vous appelez anthropologie psychanalytique ? Auparavant, vous vous étiez plutôt occupé des rapports entre la psychanalyse et la philosophie ; Freud, la philosophie et les philosophes a été votre premier ouvrage. Donc, je souhaiterais savoir d'abord comment votre déplacement vers le domaine de l'anthropologie psychanalytique s'est-t-il produit.

Paul-Laurent Assoun : - J'ai eu un trajet d'emblée transdisciplinaire, c'està-dire, moi, je suis agrégé de philosophie au départ (c'est le concours officiel pour enseigner la philosophie). J'ai, en fait, trois thèses (pour que vous voyez ce qui se joue) : une thèse d'État de sciences politiques (Fondation Nationale des Sciences Politiques), une thèse en troisième cycle de philosophie et une habilitation à diriger des recherches en psychologie, bon. Étant philosophe, j'ai toujours eu une conception très encyclopédiste : traiter l'ensemble des concepts, et j'ai fait systématiquement de l'histoire. Étant professeur, en France, il y a la possibilité de passer des concours difficiles et sélectifs de l'École Normale Supérieure, d'être élève-professeur, ce qui m’a permis, en même temps, de rayonner un peu dans toutes les disciplines, pour avoir une conception encyclopédiste d'une certaine manière. J'ai fait sciences politiques, donc, dans cette perspective, j'en suis arrivé à la rencontre de la psychanalyse, qui d'une certaine manière a confirmé l'importance de l'interdisciplinarité. En même temps, cela m'a fait choisir définitivement l'orientation psychanalytique, voilà. Je vous rappelle ça parce que, d'une certaine façon, je suis devenu psychanalyste, et j'ai été élu à l'Université d'Amiens, mais après avoir enseigné la philosophie politique aux Pays-Bas, voilà.

Donc c'est ça le trajet, j'ai commencé par enseigner la philosophie au Lycée, et ensuite, tôt, à une trentaine d'années, j'ai été donc élu, nommé à l'Université de Nimègue (Nijmegen) [Pays-Bas]. Et j'ai même appris à cette occasion le néerlandais pour enseigner, voilà. À l'époque je voulais véritablement développer toute cette dimension transdisciplinaire. Et je suis ensuite revenu en France, à l'Université d'Amiens, et finalement à l'Université Paris 7, où j'enseigne depuis des années, depuis 1992. Enfin, voilà. Donc j'ai eu ce trajet interdisciplinaire, même international. Alors, dans cette perspective, l'anthropologie psychanalytique est pour moi un complément de la clinique. Je suis d'abord, d'abord et aussi, clinicien, voilà. Ça permet au fond de revenir au rapport entre l'inconscient et le lien social.

\section{Bruno Carignano : - D'accord.}

Paul-Laurent Assoun : - Alors, c'est dans cette perspective que l'expression anthropologie psychanalytique, c'est une sorte de registre. C'est presque un 
peu pléonasme, je dirais, voilà. Puisque la psychanalyse, le freudisme est d'emblée une théorie du politique, sinon on laisse "Totem et Tabou » de côté, "La morale civilisée et la nervosité moderne » de côté, "Malaise dans la culture ", "L'avenir d'une illusion », non. Mon diagnostic, c'est presque : les analystes n'ont peut-être pas été à la hauteur en général de l'héritage freudien dans le domaine du lien social. Et encore une fois, c'est très important, c'est ça que j'ai fait, montrer dans mon manifeste Freud et les sciences sociales, soustitre : Psychanalyse et théorie de la culture, qu'on n'a pas besoin au fond d'une rallonge de l'inconscient individuel sur la forme de la sociologie. Ça ne veut pas dire que la sociologie ne nous apporte rien. Freud a l'ambition, je dis bien, de le démontrer dans la réalité. Il a véritablement l'ambition de tirer de l'inconscient une théorie du collectif, qui n'est surtout pas une théorie de l'inconscient collectif jungien, mais le sujet de l'inconscient, ipso facto, est le sujet du collectif, voilà.

Donc, c'est dans cette perspective que j'avais créé à l'époque, avec Markos Zafiropoulos, une unité mixte de recherche associée avec le CNRS [Centre National de la Recherche Scientifique]. Il faut quand même le dire : c'est nous qui avions d'abord introduit la psychanalyse au CNRS, et aussi cette discipline de l'anthropologie psychanalytique. J'ai obtenu un diplôme, un DEA, c'est-à-dire un Diplôme d'Études Approfondies, dans cette spécialité de l'anthropologie psychanalytique, qui a été reconnue à l'intérieur de la seizième section, c'est-à-dire Psychologie et Psychopathologie, voilà. Donc, je suis un peu un militant — je dis « un militant» pas du tout au sens politique, mais parce qu'il faut quand même se battre sur toutes les frontières, n'est-ce pas ? - de la théorie du collectif et de la clinique pour montrer à quel point on a besoin d'une théorie de la culture. Et l'une des ambitions de l'équipe de recherche et de ma recherche personnelle, c'était d'écrire, de réécrire "Malaise dans la culture " de Freud, évidemment l'eau a coulé sous le pont, comme on dit en français, depuis 1930, depuis l'apport freudien. Il y a des mutations, mais voilà, d'une certaine façon, il faut que la psychanalyse descende sur le terrain du collectif en permanence, non pas comme complément, mais comme accomplissement de sa vocation clinique. D'où l'importance d'un certain nombre d'objets socio-cliniques au fond.

Maintenant, que je suis Professeur émérite depuis 2017 (depuis deux ans) je reste encore à Paris 7 parce que je continue à encadrer des thèses, au moins pour six ans. J'ai aussi un séminaire doctoral, mais aussi, peut-être surtout du point de vue de l'anthropologie psychanalytique, un séminaire à la Maison Suger — qui est une annexe de la Maison des Sciences de l'Homme -, sur cette dimension-là, où je travaille avec des gens d'autres disciplines. Par exemple, actuellement je travaille sur la question du transhumanisme à l'épreuve de la psychanalyse, voilà. Il s'agit d'un phénomène idéologique particulièrement intéressant, voilà.

Bruno Carignano : - D'accord. Donc, d'après ce que vous dites, on ne pourrait pas considérer l'anthropologie psychanalytique comme un champ de savoir autonome, n'est-ce pas?

Paul-Laurent Assoun : - Voilà, c'est le mouvement même d'expansion de la psychanalyse. Mais c'est une rubrique, 
même sur forme de pléonasme, il faut insister. Vous voyez, il y a quelque chose de curieux, Lacan avait réfuté dans son séminaire D'un Autre à l'autre l'idée d'anthropologie psychanalytique, en tant qu'on pourrait lui imputer ça. Mais à l'époque on pensait, sous le terme d'anthropologie psychanalytique, à Devereux, à Roheims, à des gens qui ne sont pas dans l'orbite de Lacan. Moi, je suis d'accord avec Lacan si l'on entend par là une sorte de sociologisation ou psychologisation du social, ce n'est pas ça qu'on fait. Mais bon, c'est une rubrique universitaire.

Moi, j'aime beaucoup l'Université. Je dis ça parce qu'on pourrait penser que l'Université, c'est une structure secondaire par rapport à la recherche pure, etcétéra. L'Université est très importante pour la socialisation du travail et que la psychanalyse y soit, ce qui devient de plus en plus difficile. La situation française n'est pas idéale du point de vue de l'expansion de la psychanalyse. Paris 7 a toujours été un bastion du moins jusque là de cette dimension-là, et bon, on cherche à le maintenir. Mais objectivement, et sans prétention, l'expansion de ma recherche a un certain renom, mais aussi le fait que les ouvrages connaissent de multiples éditions. C'est très, très important ça, parce qu'un livre, c'est à partir du moment où il est diffusé qu'il continue à vivre. Ça montre qu'il y a un intérêt, ce qui est très important de ce point de vue-là. Et l'avenir de la psychanalyse dépend vraiment de la position qu'elle prendra dans le collectif. Il ne s'agit pas seulement de se convaincre entre analystes, hein ? voilà. Mais c'est une question très difficile, comment est-ce que la psychanalyse peut intervenir comme analyse critique du réel collectif ?
La politique est extrêmement mouvante, mais par contre, il y a une continuité, et moi, je suis profondément convaincu de la fécondité de l'apport freudien à la question du collectif, sans pour autant renier notre position de cliniciens, puisqu'on a affaire à ce que j'appelle le petit sujet. C'est vraiment une relation microsociale, enfin il y a un seul, une analyse ça se fait à un seul avec l'analyste. C'est un duo, n'estce pas ? Mais, en même temps, c'est le malaise dans la culture qui s'allonge sur le divan, parce que le sujet apporte ce que, d'un point de vue marxiste, on appellerait la contradiction sociale. Alors, c'est vrai que, à un certain moment, au tout début, j'avais beaucoup travaillé sur Marx, j'avais travaillé sur l'École de Francfort.

Alors, c'est vrai que j'ai une passion pour l'écriture, bon, ça m'étonne moimême parfois d'avoir fait tout ce travail, mais je pense qu'il y a là un désir de recherche qui est très fort. J'ai actuellement une quarantaine d'ouvrages, plus de six-cents articles. Parfois, c'est vrai, on me demande où est-ce que j'ai trouvé ce temps. Mais parce que, pour moi, notamment la formation, l'enseignement, n'ont jamais été une façon de perdre du temps par rapport à la recherche. Au contraire, le chercheur est profondément solitaire, même si on a quelques équipes, l'objet est quand même extrêmement personnalisé, et le fait d'avoir un public à qui on peut transmettre, ça permet... C'est formidable, en psychanalyse, on redécouvre sans arrêt en transmettant. On s'apprend en transmettant. Ce qui fait qu'en général ce que j'ai publié, c'était en rapport avec ce que j'avais dit. Pas nécessairement, ce sont des choses tout à fait différentes, c'est l'oral et l'écrit, hein ? Mais il y a une continuité 
forte de mon projet, ce qui fait que quand on est chercheur on est toujours au début. On peut capitaliser des publications, etcétéra, mais surtout pas toute sa vie répéter sa thèse. La thèse est un moment et c'est ça qui fait que, bon, les divers séminaires ont toujours été très vivants.

Bruno Carignano : - D'accord, de ce point de vue que vous venez de développer, est-ce qu'on pourrait faire une différence entre une clinique du social et une clinique du sujet individuel ? Cette différence, est-elle acceptable pour vous ?

Paul-Laurent Assoun : - Le sujet du collectif, il n'existe pas au sens où l'on pourrait l'hypostasier, le substantifier. C'est l'erreur historique de Jung d'une certaine manière, pour des raisons un peu curieuses - Il était d'ailleurs dans une problématique psychotique, mais peu importe, ce n'est pas un argument en soi. Mais c'est quand même l'idée d'un surmoi collectif qu'il s'agirait d'analyser. C'est le moment social du symptôme. Le sujet apporte la contradiction sociale sur le divan. Pas au sens d'un conditionnement de l'inconscient par l'environnement social. Ce n'est pas simplement le contexte social du symptôme - ce qui est déjà assez important. C'est le fait qu'il y a des symptômes sociaux. Et au fond Freud, qu'est-ce qu'il fait Freud?

En allant très vite : il commence par «La morale sexuelle civilisée et la nervosité moderne ", pour montrer que le névrosé n'est pas simplement quelqu'un qui a des symptômes, mais qui est un symptôme de la culture, puisque toute culture essaye de résoudre le problème de la régulation pulsionnelle, voilà. Ça, c'est un point extrêmement important, avec des très belles formules de Freud, 1908, « La morale sexuelle civilisée et la nervosité moderne ", à savoir que la culture est fondée et bâtie (aufgebaut, ça veut dire fondée) sur la répression pulsionnelle. (Pas au sens de Reich, hein, qui a une théorie freudomarxiste d'une certaine manière, au moins au début). Ce qui veut dire que le sujet qui a des problèmes profondément personnels et singuliers de répression pulsionnelle, ça devient un dissident de la culture. C'est curieux de le dire comme ça, mais voilà, c'est un dissident de la culture. Plus exactement: la névrose, où qu'elle se porte, quelle que soit la forme qu'elle prend - je résume une formule de Freud - , la névrose sait déjouer, rendre vaine l'intention de la culture. Qu'est-ce que ça veut dire ? Ça veut dire que la culture a tendance à vouloir réguler, c'est normal. La culture, c'est autre chose que la psychopathologie, mais, en même temps, il y a une résistance intrinsèque du sujet, par son symptôme, à la culture en tant que telle. Or, qu'est-ce que les analystes ont fait de ça ? Il me semble que peut-être ils ont perdu de vue, par une psychopathologisation nécessaire des petits sujets uniques, cette fonction du sujet névrosé, voilà.

Alors, ensuite, très rapidement, mais c'est intéressant de voir les étapes. Il y a cinq étapes chez Freud. 1908, il repère ça : le sexuel est un symptôme social fondamental de dissidence. Ça n'a rien à voir avec la sexologie, voilà. Du coup, il va jusqu'à faire une généalogie de la culture avec "Totem et Tabou ", bon, avec ce mythe du père qui est nécessaire pour comprendre cette prise du symptôme dans l'origine même, même si c'est une histoire évidemment fictive, mais qui permet de comprendre que l'enfant phobique ou le névrosé obsessionnel adulte sont en- 
fin des véritables expressions, en quelque sorte, de cette contradiction culturelle. Ensuite, sans arrêt, avec cette question du retour du père, il tombe sur "La psychologie collective et l'analyse du moi ", où il fait une théorie des institutions. Il n'appelle pas ça des institutions. Il appelle ça des foules artificielles. Enfin, bon, c'est ça que je dis dans Freud et les sciences sociales, voilà. Ensuite, il débouche sur "L'avenir d'une illusion » (1920, "Psychologie collective », 21 exactement). Ensuite, 1927, c'est "L'avenir d'une illusion ", dont nous avons fait quand même la première édition critique au plan international, avec des notes, etcétéra, aux Éditions du Cerf. Je le signale parce que c'est un texte qui n'a pas toujours été remarqué, voilà. Et on en arrive à " Malaise dans la culture ", où il introduit la pulsion de mort. Vous remarquerez que chaque émergence de la métapsychologie se manifeste par une mutation de la théorie du collectif.

On commence avec la libido, on passe par le narcissisme, et la pulsion de mort là joue un rôle absolument essentiel, voilà. Puis évidemment, "L'homme Moïse et la religion monothéiste ». Pourquoi estce que Freud, qui se présente comme un athée, sans état d'âme, il s'intéresse tellement à la religion ? C'est parce que c'est dans la religion, et notamment dans la religion historique, qu'on peut voir le travail du désir dans le collectif, voilà. Donc, ces éléments, ils pourraient être plus ou moins connus, mais je crois que l'originalité, c'est d'en faire encore plus. Quand j'ai écrit Freud et les sciences sociales, j'ai été frappé encore plus de l'énormité du corpus freudien sur le social. Alors, bien sûr, il y a là une question très importante du rapport entre l'idéologie et la psychanalyse. Si on ne fait pas ça, la psychanalyse peut, com- me ça a eu lieu aux États-Unis ou ailleurs, devenir une idéologie de la psychologie du moi, voilà. Donc c'est quelque chose qui n'est pas simplement intéressant, qui est nécessaire et qui a affaire quand même avec l'avenir de la psychanalyse. Parce qu'on peut se demander si à un certain moment..., Lacan lui-même dit : qu'il n'y avait pas de nécessité que la psychanalyse existe, il n'y a pas de nécessité qu'elle vive et qu'elle dure. Je pense que la psychanalyse a une position extrêmement forte si on reste à la hauteur de l'exigence freudienne.

Enfin, j'ai fait un Dictionnaire des øuvres psychanalytiques, où je parle de tous les psychanalystes, pour mieux transmettre. C'est incroyable la désinvolture avec laquelle certains évoquent Freud sans le lire, sans voir qu'est-ce qu'il dit, à quel moment de sa démonstration. Mais nous ne sommes pas condamnés à répéter Freud. Freud nous fait travailler, il fait vivre notre recherche. Donc, de ce point de vue-là, bon, moi, je découvre. Je vis avec les textes freudiens depuis des années. Pourtant, je suis tout à fait étonné des richesses qu'il y a dans les textes freudiens, qui nous parlent toujours. C'est-à-dire, de ce point de vue-là Freud n'est absolument pas mort, et ce n'est pas l'idéalisation de quelqu'un. Ça renvoie à cette question, à ce côté un petit peu étonnant, voire miraculeux : c'est bien remarquable, dit Lacan, qu'un homme seul, à un certain moment, ait pu inscrire un champ social, un champ scientifique nouveau, une science singulière.

Il y a des gens qui veulent être, comment dire ? qui veulent avoir une petite originalité à tout prix. Moi, ce qui me parait intéressant, ce qui est très ambitieux à mon sens, c'est d'être à la hauteur du geste freudien, voilà. Sur la route, bien sûr, j'ai rencontré Lacan, mais Lacan a une autre 
stratégie. Lacan homologue une mutation épistémologique, on se retrouve avec des signifiants, avec tout ce que Lacan a fait. Mais il ne faut pas que les lacaniens oublient cette évidence : que Lacan n'a pas été possible sans Freud, ce que Lacan lui-même n'a jamais oublié. Ce qui m'a toujours passionné, quand j'ai enseigné à Paris 8, c'est de reprendre chaque concept de Lacan à partir d'un concept freudien. Et c'est aussi ce que j'ai développé dans mon Que sais-je ? Lacan, qui est un livre de poche, où j'avais essayé comme ça, dans un volume limité, d'initier le public à cette systématicité de la démarche lacanienne. Disons que pour moi — nous sommes nombreux à travailler sur Freud et Lacan mais pour moi le transfert majeur est freudien, voilà. Par contre, j'ai une réelle admiration pour Lacan en tant qu'il a tenté, contre une sorte d'opérationnalisation du savoir analytique, du savoir freudien, de revenir au geste de fondation, voilà. Donc le retour à Freud me parait essentiel. C'est dans le sens du retour à Freud que je me propose de continuer.

Bruno Carignano : - Donc vous avez bien montré que l'approche psychanalytique implique en soi quelque chose d'anthropologique, mais qu'elle suppose, en outre, un au-delà, un plus, si l'on peut dire, qui n'est pas présent dans l'anthropologie toute seule, n'est-ce pas ? Par rapport à cela, je souhaiterais vous demander, pour bien saisir dans chacun des cas la différence des approches... Bon, il y a un petit paragraphe dans l'ouvrage du psychanalyste Moustapha Safouan qui s'appelle Études sur l'Edipe. Là il ébauche une distinction que je souhaiterais que vous commentiez. Donc je souhaiterais, si vous êtes d'accord, vous lire le paragraphe en question pour vous demander comment on pourrait penser la distinction que l'auteur propose du point de vue de l'anthropologie psychanalytique. Il s'agit du paragraphe suivant :

Cette conception indienne - puisque Lévi-Strauss nous dit la tenir de la bouche des Indiens - de la loi de l'interdiction de l'inceste comme une loi d'échange, si elle suffit à expliquer l'exogamie, n'explique pas l'interdiction de l'inceste. Il n'y a rien, dans la perspective de cette théorie qui empêcherait, par exemple, qu'on confie l'initiation du jeune garçon à sa mère avant de le lancer sur le marché des hommes mariables ou encore de preneur des femmes : on reconnaît là un fantasme assez typique de l'obsessionnel' ${ }^{1}$.

Quelques lignes plus bas, il arrive à en déduire la distinction entre la femme comme objet d'échange et la femme comme objet de désir. Quel est votre avis au sujet de cette distinction faite par Safouan?

Paul-Laurent Assoun : - Distinction entre donc ?

Bruno Carignano : - Donc, entre l'approche de la femme comme objet d'échange et la femme comme objet de désir, dans ce sens précis : l'anthropologie pourrait expliquer l'exogamie, mais l'interdiction de l'inceste n'en découle pas nécessairement. C'est-à-dire, dans ce sens, l'interdiction de l'inceste serait plutôt quelque chose de strictement lié à la psychanalyse.

Paul-Laurent Assoun : - Oui, tout à fait. Mais c'est une mise au point utile. On sait tout le rapport entre Lacan et Levi-Strauss, hein ? Mais, contrairement 
à la théorie ethnologique qui en fait un instrument de régulation - l'échange des femmes -, là on est quand même dans l'irréductible du sexuel, voilà. Ça, c'est un point important, l'un des rameaux de ma recherche, que j'ai commencé en 83 , autour de Freud et la femme.

Quand Freud dit que ce que veut la femme est la seule question laissée sans réponse par la psychanalyse - il le dit à Marie Bonaparte -, d'une certaine manière, ça incite à le relire dans le contexte de ce que vous dites. C'est-à-dire qu'il y a, pour ainsi dire, quelque chose d'irréductible dans la question du féminin, quelque chose qui à la fois défie la culture, où la femme ne serait pas simplement un bien sexuel qui circulerait. Mais c'est une idée freudienne, d'ailleurs, que la femme pose la base, les femmes sont les porteuses des intérêts sexuels de l'humanité. On leur met sur le dos la sexualité, mais non pas leur désir à elles. Ça pourrait être des idéologies politiques anciennes et contemporaines. D'ailleurs, ce n'est pas ça du tout ce qu'il veut dire. C'est-à-dire, n'est-ce pas ? le sexuel est en partie au sexe féminin pour poser une question structurelle, structurale, en un sens différent du structuralisme lévistraussien. Mais il y a une affinité avec cette question du caractère au fond le plus stupéfiant qui soit, anthropologiquement parlant, c'est-à-dire le rapport entre un homme et une femme, voilà. Ça, c'est un point extrêmement important.

C'est d'ailleurs pour ça que je reviens à ce que je vous disais tout à l'heure : que Lacan n'aimait pas trop le terme d'anthropologie, parce qu'il supposerait un anthropos non divisé, alors que, utile rappel de Lacan, l'anthropos est foncièrement divisé. Ça n'a rien à voir avec la sexologie, qui considère ça comme une fonction, ou la biologie. Non, c'est le fait qu'il y a ce quelque chose qui fait chroniquement symptôme, qui est le sexuel. Donc, l'analyste ne devrait pas ouvrir la bouche sans parler de la question du sexuel et de la castration. Il n'est pas là pour reproduire les idéaux sociaux ou les humaniser. Non, d'abord, penser ce réel-là qui revient - voyez comment moi, je circule entre Freud, Lacan et Lévi-Strauss c'est-à-dire l'impossible du rapport sexuel, voilà. Freud ne l'a jamais dit comme ça. Mais il a dit plus modestement qu'il semble y avoir quelque chose de non favorable à la satisfaction dans la pulsion sexuelle. Il est quelqu'un qui parle tout le temps du sexuel pour dire qu'il y a vraiment un truc là problématique. Et c'est là qu'il met d'une certaine manière le symbolique. $\mathrm{Ce}$ qui fait qu'à chaque couple, si je puis dire - la manière la plus directe de le dire - à chaque couple qui se forme entre un homme et une femme se repose la question de l'anthropologie, voilà. Donc ce n'est pas une fonction parmi d'autres. Pour répondre aux gens qui disent : " mais pourquoi on nous bassine tellement avec la sexualité ? C'est une fonction importante, mais c'est une fonction parmi d'autres ". Non ! C'est le point de révélation du symptôme.

Et ce n'est pas par hasard s'il y a tout un révisionnisme psychopathologique qui fait que maintenant on parle - on va y revenir avec votre question - des borderlines. On a remplacé la structure par l'organisation et la désorganisation psychique. Donc tout est à refaire. L'un des points qui me frappe beaucoup, c'est quand même ce geste freudien qui fait qu'il y ait tout à refaire. Parce qu'à la fois la psychanalyse a un certain prestige - évidemment ce n'est pas à un argentin que je dois expliquer ça, vous avez un rapport tout particulier 
à la psychanalyse. De façon générale, il y a aussi un prestige en France de la psychanalyse, mais par contre il y a une résistance structurale au vif de ce que dit la psychanalyse. On veut bien qu'on nous titille avec notre inconscient, nous flatte, etcétéra. Par contre, le public, en lui disant qu'il a quelque chose de l'ordre de la castration, ça ne peut pas être populaire.

C'est pour ça que Freud, à un certain moment, dit à Binswanger cette phrase : « il semble qu'il n'y a rien en soi moins favorable à l'organisation humaine que ce que la psychanalyse lui annonce ", voilà. Ce n'est pas une idée un peu de coquetterie qu'on prend : " on résiste ». Ce n'est pas ça, c'est qu'il faut toujours prendre le savoir de l'homme à rebrousse-poil. Il n'y a même pas de science de l'homme, parce que c'est plutôt la science de ce qui manque à l'homme, cette fameuse formule, voilà. Donc tout ça, ça implique ce rôle absolument central. Du coup, toute la question du féminisme se replacerait, qui fait que la femme en a quand même spécialement bavée de cette question-là. Elle a été réprimée, pas simplement réprimée, si je puis dire, pour des raisons idéologiques, mais parce qu'il y a quelque chose qui angoisse dans la femme, voilà, qui fait qu'elle représente ce sexuel. Donc, la psychanalyse est extrêmement critique, c'est un élément très important de critique de la condition qui a été faite à la femme. Mais comme la psychanalyse ne produit pas de vision de monde, en termes plus modernes, d'idéologie, ça n'arrange personne. C'està-dire que les pensées conservatrices et progressistes sont embarrassées de ce qui a affaire au réel psychanalytique.

Ce qui me passionne chez Freud, c'est le désir du réel. Mais la psychanalyse ne peut pas être autre chose qu'un savoir critique. En ce sens : elle ne peut pas être conservatrice, voilà. Alors, maintenant il y a toujours cette idée que..., comme l'idéal social à besoin de choses quand même optimistes, alors que la psychanalyse n'est ni optimiste ni pessimiste - la pulsion de mort n'est pas du tout un concept pessimiste, c'est la structure du réel psychique, voilà. Donc, à partir de ce moment-là, on invente - et c'est ça que je travaille dans mon séminaire dans la Maison des Sciences de l'Homme - , toutes sortes de bouche-trous à la mode : la résilience, le burnout (c'est une notion intéressante, mais il faut voir pourquoi le travail est tellement sujet au symptôme). C'est-àdire des indices d'un discours social qui veut résorber la question de la castration, parce qu'à chaque fois c'est ça. La résilience, c'est beaucoup plus sympathique, puisqu'il faut faire du positif avec du négatif, etcétéra. Mais ce n'est pas ça. Évidemment que la psychanalyse veut que le lien social aille mieux, mais cela suppose d'affronter le réel dans son caractère hard, si je puis dire, voilà. Bon, il y aurait beaucoup plus à dire sur la structure.

Bruno Carignano : - Mais je crois qu'on pourrait reprendre cela du point de vue de la problématique des états limites.

$$
\text { Paul-Laurent Assoun : - Exacte- }
$$
ment.

Bruno Carignano : - Il s'agit de quelque chose qui se rapproche beaucoup de ce que vous venez de dire. Donc, par rapport à cela, comment peut-on envisager la catégorie clinique des états limites ou des borderlines, tellement répandue dans la psychanalyse française contemporaine ? Comment peut-on lire ce phénomène du 
point de vue de l'anthropologie psychanalytique ? Pourquoi cette catégorie est tellement répandue ? Quelles sont les raisons?

Paul-Laurent Assoun : - Ça, c'est une question qui m'est chère, vraiment. J'avais fait un article pour un colloque d'Espace Analytique sur un certain vous l'avez indiqué - style borderline de la modernité, voilà. Qu'est-ce que je veux dire ? Je veux dire que dès lors que névroses, psychoses et perversions - là je ne vais pas réciter la psychanalyse, mais montrer rapidement ce qui est important -, on n'a pas été fichu, dit Lacan, de créer une nouvelle perversion [rires]. Bon, on n'a pas trouvé de tierce structure. C'est ce que j'appelle des politiques de la castration. La névrose avec le refoulement, qui est l'opérateur, qui est le mécanisme de défense, plus que le mécanisme de défense, le jugement fondamental de la structure névrotique. Là alors, chez Freud, il y a un problème. Pour le refoulement au sens psychotique, Lacan propose la forclusion. J'en suis d'accord, cela explique beaucoup de choses dans le psychotique. Et puis le déni, la Verleugnung, le déni pervers. Vous avez, à chaque fois, des politiques de la castration. La castration, c'est pour tout le monde, et évidemment, il y a des dialectes pour chacune des structures. Ne croyons pas comme certains que la structure arrange les choses, et que ça permet de ranger les gens dans un tiroir, au contraire. Ça nous embarrasse, la structure, mais si on ne passe pas par la structure, qu'est-ce qu'on va faire ? On va supprimer, c'est ce que je viens de dire, l'embarras du sexuel, voilà. Freud a toujours dit que quiconque débarrassera l'homme de la servitude se- xuelle sera bien accueilli, Jung et les autres, voilà. Mais ça continue, ça continue !

Bruno Carignano : - Oui, tout à fait.

Paul-Laurent Assoun: - C'est le point de résistance. Donc, on s'est rapatrié, si je peux le dire, en supprimant le sexuel, la castration, du côté d'une désorganisation psychique. Et actuellement, ce qui a du succès en France, ce qui a le plus de succès, c'est quoi ? Justement, toutes les personnalités hybrides. Il y a un texte, d'ailleurs intéressant, de Helene Deutsch : "Les personnalités comme si ». Je dis parfois : des personnalités comme ci, comme ça, avec des faux-selfs. Et si je peux dire, le standard, la vedette, c'est le borderline. Les borderlines, ils sont nés de la psychiatrie. Il y a un auteur oublié, Mercadier, un psychiatre qui fait une histoire des borderlines dans les années 70, qui est intéressant à relire. Il rappelle que ce sont les psychiatres qui ont inventé le borderline, notamment un certain Hugues, un psychiatre anglo-saxon combattant Kraepelin (qui sépare la nosographie), pour montrer qu'il y a des entités mixtes. Il avait repéré des sujets borderlines qui ont un certain rapport à la folie : ils sont fous par intermittence et franchissent une ligne, voilà.

Ensuite, le terme a été utilisé par Eisenstein par exemple, peu après la Seconde Guerre mondiale, notamment, pour ces sujets qu'il veut repérer. Parce que si on se trompe en prenant un névrosé pour un psychotique ou vice versa, on risque de se trouver dans des situations de traitements psychiques catastrophiques. Et finalement, il crée l'idée que ce n'est ni l'un ni l'autre, donc des borderlines. Bon, 
je dis parfois - c'est peut-être un peu facile de le dire comme ça -, si les borderlines existent, je voudrais bien savoir qu'est-ce que c'est ce sujet qui passe la moitié du temps dans la névrose et l'autre moitié dans la psychose. Ce n'est pas possible, on ne peut pas être un psychotique intermittent, voilà.

Par contre, juste par contre - je vais jusqu'au bout - , oui, la description qu'on fait des borderlines correspond à une certaine réalité. Parfois, il m'arrive de voir des patients qui ont les traits. Ils ont ces traits, bon, quand ils sont passés comme ça au scanner psychiatrique, ils ont ces traits. Alors, mon idée - regardez, votre question est intéressante parce qu'elle est très cohérente - mon idée, c'est que c'est une fausse entité psychopathologique, mais un vrai symptôme social subjectivé, qui donne un style borderline au sujet du malaise contemporain. Et, du coup, c'est ce qui fait que les borderlines ont un succès, un gros succès. Par exemple, tous ces faux concepts - burnout, résilience - c'est le repérage des vrais symptômes. Donc, au fond, ce savoir-là, ce savoir social, il travaille pour nous, mais dans notre recherche, on descend sur le terrain du sujet pour comprendre effectivement comment cela se passe. Après tout, les borderlines borderline, c'est presque un descriptif social - , ce sont des gens qui sont des intérimaires permanents, et pas seulement parce que le formage est là, c'est la vraie réalité. Mais, en même temps, c'est parce que ces sujets-là ont toujours des grandes difficultés dans la continuité du désir. Disons, pour aller vite, la jouissance l'emporte sur le désir. Ce sont des sujets qui sont alors bâclés ; la névrose d'abandon (Germaine Guex), qui date un peu, voilà.
En clinique tout est ouvert, il faudrait discuter de tout, donc on ne va pas se réfugier dans la structure. Mais il faut démontrer la fécondité clinique des structures qui fait qu'on crée des entités imaginaires, mais qui soutiennent l'imaginaire social, voilà. C'est pour ça que je suis gêné quand des analystes adoptent ces entités psychopathologiques en y injectant une dose freudienne. Non, il y des raisons profondes pour lesquelles ni Freud ni Lacan n'ont eu besoin des borderlines. Mais naturellement, chaque fois qu'on hésite... Alors voyez, il y a des sujets qui nous mettent dans tous nos états, vraiment on hésite, on ne peut pas trancher. Dès les entretiens préliminaires on sent bien un problème, mais ça peut durer des mois, on ne va pas trancher aussi facilement, hein ? Mais c'est parce que nous sommes nous-mêmes limites à un certain moment dans le diagnostic, que nous allégeons - enfin, ceux qui font ça - par le jugement : "ce sont des états limites ». Non! Alors, ça ne renvoie pas simplement au déclin du père, qui est un terme ambigu, mais à cette question de la renégociation avec le symbolique.

$\mathrm{Ne}$ disons pas que le père n'a plus la cote. Il y a un grand changement des idéaux et des identifications dans la structure familiale, c'est une question pour nous : anthropologie psychanalytique, voilà. Ça, c'est intéressant, mais les sujets les plus désespérés de la postmodernité, ce sont tous ces sujets errants borderlines. $\mathrm{Si}$ on nous bassinerait un peu moins avec les postmodernes... : «bah, tout change, tout change, tout le temps, les postmodernes, les hypermodernes, etcétéra... ». Bon, ce sont des sujets qui ont une passion du père désespérée. Qui est-ce qui cherche le plus 
le père ? (Ce qui fait qu'ils vont le trouver). Mais comme les religions historiques sont dans une position aussi, comment dire ? branlante ; en même temps que le père symbolique, qu'est-ce qu'ils vont trouver? Des sectes. Et dans les sectes, qu'est-ce qu'ils vont trouver comme gourou ? Ou bien des psychotiques stabilisés - qui se stabilisent au moyen de leurs sectes - ou bien des pervers, voilà. Mais après on se dit, en se révérant, c'est incroyable : " j'ai tout donné, mon carnet de chèques, etcétéra ". C'est justement qu'à un certain moment ils se sont soignés de leurs malheurs du côté du père au moyen de leur gourou, ce qui n'est évidemment pas recommandé.

Qu'est-ce que dit l'humanisme ? Et bien, c'est une violation de la pensée. Mais non ! C'est un symptôme. Le fait que les sectes pullulent montre qu'est-ce que c'est, non pas le déclin en soi du père, mais toute cette crise qui fait que vous allez trouver, par exemple, dans les borderlines - je reviens à la question clinique - , des psychotiques pas encore déclenchés, ou qui ont stabilisé selon des manières très particulières une psychose. Vous allez trouver des névrosés dans des situations justement limites de réalité, hein ? L'obsessionnel, il est limite par moments, mais ce n'est pas pourtant un état limite, voilà. Ce qui fait que c'est comme une population. C'est curieux, ce qui me vient, c'est un peu une image, effectivement sur ce que certains appellent la postmodernité : un aéroport, engorgé de gens qui n'ont pas leurs papiers, qui ne peuvent pas décoller, voyez ce que je veux dire. Et, du coup, je garde la notion de borderline, moi, pour nommer un style. Mais si l'on en fait une structure psychopathologique, c'est catastrophique.

C'est le fait que le sujet semble désabonné du sexuel. Et, du coup, on a une vieil- lerie psychologique, qu'il faut bien dire, qui est l'organisation psychique. L'organisation psychique n'est pas une mauvaise notion pour décrire. Moi, ma grande idée, c'est que quand on décrit, ce n'est pas grave, mais si on décrit en croyant expliquer, c'est catastrophique. D'où mon recours à la métapsychologie, qui est, à mon gré, à mon goût, la seule théorie consistante pour aborder la psyché via l'inconscient. Et d'où, quand même, la pauvreté de ces modèles théoriques, qui est compensée par le talent de ses utilisateurs. Donc voyez dans quelle situation on est, c'està-dire chacun a sa petite province, alors que là les richesses freudiennes sont sousutilisées. Mais ce n'est pas par, j’y insiste parce que c'est très important, pas par souci de se réfugier chez Freud, de s'accrocher à la théorie freudienne, mais parce que Freud est encore à découvrir, encore faut-il penser à l'utiliser là où il faut.

Et, du coup, voyez, je me retrouve, quand même, face à des analystes qui ont méconnu le social. Cela fait que souvent, certains ont fini par compléter la psychanalyse, comme théorie irremplaçable du réel, par des prêts-à-porter des visions du monde, de tous bords, voyez! Alors, c'est vrai que ça amène à penser à une certaine atopie de la psychanalyse, qui intervient toujours de l'extérieur. Mais justement, si elle a les moyens de penser à la contradiction sociale via l'inconscient, elle peut habiter la condition contemporaine. Vous connaissez la formule de Lacan qui dit que l'analyste doit être à la hauteur de la subjectivité de son époque. Une formule quand même un peu cryptée. Il dit, plus précisément : dans son acte. C'est-àdire un analyste ne peut opérer dans son acte, dans sa praxis analytique, comme thérapeute, pas simplement psychothéra- 
peute... (je n'ai rien contre ce terme, je ne veux pas être puriste, mais très vite ça peut filer vers l'imaginaire). Alors, qu'est-ce qu'il veut dire ? Il faudrait parler longtemps de cette formule. Il veut dire qu'effectivement l'analyste est toujours impliqué dans son époque, qu'il est de son temps. Mais pas de son temps au sens de vitrine de l'époque, de buzz informatique, de Facebook, enfin.

Il ne s'agit pas d'être grognon, de dire : « tout ça, ça change, c'est nul ». Non. Vous voyez ce que ça fait : ce tas des petits individus sans appartenance qui se regroupent, qui veulent faire..., je ne suis pas en train de dire qu'il faut interdire Facebook. Juste, qu'est-ce qui fait symptôme ? Vous avez des sujets qui sont tout heureux qu'il se fasse un petit attroupement autour d'eux. Et même le statut évidemment de la sexualité : chacun a un Facebook, mais il n'est pas là. Les sites de rencontres, je n'ai rien contre les sites de rencontres - parce que, d'ailleurs, ça peut être très intéressant pour certains patients un site de rencontre - , mais c'est quand même le meilleur moyen d'éviter la rencontre. Au sens de la rencontre, dans ce cas-là, du réel. Très souvent, j'ai remarqué que certaines patientes ont été tellement déçues par les hommes qu'elles les mettent en série, pour voir un peu qu'est-ce qu'ils veulent, parce que "qu'est-ce que veulent les hommes ?", c'est une question que se posent aussi les femmes. C'est incroyable, même la pornographie, le rapport qu'il y a entre la jouissance et la.... Avec le sexuel, il faut se taper la division du sujet, ces corps qui se frottent, qui se pénètrent. Ça soulage de la contradiction.

C'est comme ça que je comprends aussi qu'un homme - un obsessionnel par exemple, qui a beaucoup de mal avec la question de l'amour, avec la question de faire tenir un couple, etcétéra - il va se distraire avec des formes substitutives, là où il est allégé de la culpabilité du désir. Alors, « ah ! culpabilité, c'est le retour de la religion ». Évidemment pas. La culpabilité en psychanalyse est un symptôme. Ce n'est pas ce qu'on cherche, ce n'est pas un but, c'est un effet. Alors, il faut être très clair sur cette question-là. À partir de là, on a un levier extrêmement important. Donc on s'intéresse à tout ce qui est nouveau, mais on n'est pas philonéiste parce que, vous savez, misonéiste, c'est celui qui n'aime pas les nouveautés Mais il y a des philonéistes qui nous disent tout le temps : "ça le fait, c'est la mode, ça change, ça change, ça change ». Alors, naturellement la psychanalyse has been. C'est pour ça que je travaille sur le transhumanisme.

Parfois, on me dit : « est-ce que le transhumanisme mérite tellement qu'on s'y intéresse ? Parce que c'est véritablement une idéologie gigantesque ». Oui, il mérite qu'on s'y intéresse parce que c'est un symptôme récapitulatif, voyez. L'homme augmenté, pourquoi est-ce qu'il est populaire ? Parce qu'il dépasse la castration, compte tenue de l'évolution scientifique, qui est énorme - il faut considérer comme un progrès gigantesque l'évolution scientifique - Mais ils disent : " comme ça on va pouvoir..., il viendra un moment où il n'y aura plus de souffrance dans l'humanité, il n'y aura plus d'handicap. Même, peut-être, on pourra opérer tous les gens à la naissance pour leur donner un nouveau corps cyborgien ". D'un côté, certes, c'est assez délirant, mais de l'autre, ça s'appuie sur la science et la chirurgie. D'où toute cette question évidemment de la science en tant qu'elle vient équiper le désir. 
Regardez la question - je vais aller très vite sur la question du sexuel — : le transsexuel, il y a un problème en effet d'identité, pour ne pas appartenir au sexe qui convient. Mais, du coup, la science devient une loi. Et vous avez, par exemple, telle adolescente qui a un problème d'identification familiale, qui va venir dire à son psychologue ou sa psychologue : «n'embêtez pas mes parents avec tout ça. Non, moi, je sais quel est mon problème : je n'ai pas le bon sexe. Donc, le meilleur que vous puissiez faire pour moi, c'est de me faire un certificat comme quoi je dois changer de prénom et me faire opérer ». La psychanalyse n'a évidemment pas à douter de la transsexualité, de la souffrance transsexuelle, mais maintenant, la loi qui a été désertée du côté du père symbolique, revient à travers la science, voilà. Ce n'est pas que le médecin soit machiavélique. Le scanner, c'est formidable comme avance.

Mais en ce moment je travaille sur les hypocondriaques. Les hypocondriaques, ce sont ceux, qu'au scanner, n'ont rien, et on a les moyens vraiment de savoir qu'ils n'ont rien, et cela n'empêche qu'il y ait un malaise dans le corps. Freud appelle ça le narcissisme, Lacan appelle ça la délocalisation de la jouissance. Donc ce truc-là qui ne se voit pas au scanner et qui vient dans le réel, c'est pour nous. Donc, on gère les déchets de la science, voyez. Mais la science, du coup, recroise la question du pouvoir. Enfin, avec tout ce que j'ai dit là, on voit bien à quel point le présent, si je peux dire, est présent dans les propos analytiques. Mais si on oublie la structure, on fait de l'idéologie, voyez.

Mais cela suppose évidemment un laboratoire de recherche qui parle des objets au fur et à mesure qu'ils apparaissent. Moi, j’avais travaillé aussi sur les épisodes, pendant la révolution française, du déterrement des rois, du fait que les révolutionnaires ont déterré tous les corps des rois, enfin. C'est un événement de ce genre qu'on constate actuellement, un événement de ce genre qui est exorbitant par rapport à la causalité historique. Vous voyez, le retour du père et de la haine du père dans le réel, je vais très vite, voilà. Donc il faut penser à mobiliser notre savoir freudien comme une théorie du réel. D'ailleurs, Lacan plus il avance, plus il s'y intéresse. Il est parti de l'imaginaire, le symbolique, et finalement il arrive au réel. Donc la théorie analytique n'est pas une théorie psychologique au sens de l'imaginaire du moi, c'est une théorie du réel et du symbolique, voilà.

Bruno Carignano : - D'accord. Donc, d'après ce que vous pensez, la catégorie des états limites reste valide au niveau de la description de l'organisation psychique ? Pas au plan de l'explication théorique, c'est ça ?

Paul-Laurent Assoun : - Donc, pas au plan théorique. Mais c'est fait pour ouvrir une discussion entre cliniciens : "voyons ce sujet ". Il faudrait dire la même chose de la psychosomatique. La psychosomatique en arrive à créer un sujet psychosomatique imaginaire. Mais par contre, ce qui est absolument essentiel, dans le rapport entre corps et symptôme, c'est évidemment le moment somatique absolument décisif de la structure, voilà ! Est-ce que c'est un psychotique, un pervers ? - un pervers fait du corps ! - Ou une conversion hystérique ? Donc c'est un moment de retour. Mais il est, quand même, aussi intéressant que les patients psychosomatiques se mettent à pulluler, 
voilà. Donc, il y a toutes ces théories des épidémies qui montrent qu'à un certain moment on trouve plein de borderlines, etcétéra. C'est un produit de discours, qui renvoie à une certaine réalité. Mais je distingue bien la réalité, qui est toujours imaginaire, qui soutient le quotidien, du réel qui vient troubler la réalité, voyez !

Bruno Carignano : - Tout à fait. Et comment est-ce qu'on pourrait penser le rapport à la structure psychique dans ces divers cas de symptômes liés au contemporain ?

Paul-Laurent Assoun : - Ben, si vous reprenez « Le malaise dans la culture »... C'est un peu triste " Le malaise dans la culture » parce que j'ai toujours à me taper, à nouveau, des malaises. Donc, chaque génération... Qu'est-ce que «la génération » veut dire ? Je vais faire quelque chose de neuf. Ça va le vieux, mais maintenant je vais tenter quelque chose. Et à la fin, on se retrouve dans certaines impasses qui font qu'entre l'homme et la femme ça cloche - certains diraient, de façon très conservatrice : "voyez, il ne faut rien changer, c'est éternel ! ". Non, la structure n'est pas éternelle. Elle est le retour permanent d'une impasse dans la structure du désir qui est nommée par le sexuel, voilà. Donc, structure psychique ; moi, je préfère dire : structure du réel inconscient. Donc, on invente des choses, on invente des dispositifs de jouissance nouveaux.

D'accord, le rapport sexuel est impossible, mais c'est au contraire excitant, « nous, on va y réussir » - deux amoureux n'ont pas l'impression que c'est impossible le rapport sexuel, hein ? Donc, l'homme et la femme réinventent les cho- ses en permanence. Mais c'est une disposition particulière. J'essaie de repérer la subjectivité analytique au fond. Finalement, un élément de conclusion, c'est peut-être que Freud..., enfin, ça serait vraiment très bête que l'humanité, à un moment donné, abandonne la tentative freudienne. Or, il y a des gens qui s'emploient à aller dans ce sens, en en faisant une vieillerie du dix-neuvième siècle. C'est le mérite de Lacan d'avoir rajeuni la problématique. Mais voilà, nous n'avons pas à adapter l'inconscient à la réalité. L'inconscient est dans le réel, donc il faut l'y retrouver, voilà ! On avait toute la théorie freudienne de la sexualité, on avait la logique de la sexuation lacanienne, et on nous refile la théorie du genre!

La théorie du genre repose des questions qui peuvent être intéressantes, sur le conditionnement social qui fait qu'on construit des garçons et des filles, etcétéra. Mais enfin, comme par hasard, qu'est-ce qui y est bravé ? Vous y repérez, toute la logique du sexuel, du psychosexuel. Quant à l'angoisse de castration, " attention à l'angoisse de castration, c'est encore un effet pervers de la doctrine du genre [sic] qui fait que le féminin passe à la trappe, etcétéra ». Et, très souvent, les théoriciens de la théorie du genre sont des militants d'une révolution.

Ce qui fait qu'une fidélité à la position freudienne est une question de fidélité au réel, voilà. Je dis souvent que Freud est quelqu'un qui a découvert la langue de l'inconscient. Très souvent, on me dit : " mais Freud n'est pas critiqué dans votre projet ». Mais pourquoi ? Vous allez critiquer l'espagnol d'être de l'espagnol ou le français d'être du français ? C'est une langue. En plus, Freud, vous le savez, il avait une passion pour l'espagnol - je 
le signale dans un texte que j'ai fait pour le numéro sur Freud dans les Cabiers de l'Herne (2015)—, quand il était adolescent, et, en fait, il ne voulait pas l'apprendre. Donc, il discutait avec Silberstein, un type d'une maturité incroyable à dix-sept ans. Ils prenaient un texte en espagnol, un texte unique - j'aimerais bien l'identifier, mais c'est un texte simplement tout à fait banal - , et ils parlaient une langue - il était un très bon élève - mais sans vouloir l'apprendre. Et donc c'était une langue qui leur permettait enfin de communiquer dans leur amitié, voilà. Et je me disais, Freud, au fond de sa passion à dixsept ans..., sa passion c'était de trouver une langue qui soit un dialecte dans lequel on lit directement - si vous commencez à feuilleter votre dictionnaire, votre vocabulaire, votre grammaire, vous l'apprenez.

Il voulait rentrer dans l'espagnol décidément. C'est incroyable, vingt ans après, il a commencé à faire ça avec la langue de l'inconscient. Et voyez, la langue de l'inconscient, ça fait plus lacanien, mais voilà, c'est ce point qui est essentiel. Donc, quand vous lisez Freud, vous apprenez la langue de l'inconscient, mais ça ne veut pas dire..., Freud a eu des désarrois à certains moments donnés, il a eu des bévues... Si vous voulez, il a fait des fautes dans sa propre langue, voilà. Mais je veux dire : avec Freud on peut discuter. Et j'ai remarqué que ceux qui ont la tête soi-disant plus ouverte - « la structure, c'est trop rigide " - , ils sont très, très rigides dans les descriptions des borderlines..., comme s'il s'agissait de les ficher : "ce sont des intérimaires ». Le savoir freudien est beaucoup plus libre. Alors, qu'est-ce qu'il est devenu dans la réalité avec la psychologie du moi ? Lacan a nettoyé les écuries d'Augias, comme on dit, il a mis de l'ordre là-dedans. Surtout, en même temps, ce qui est intéressant est, lui-même dit, que son savoir est toujours à refaire. Il dit à ses disciples : "faites attention de ne jamais considérer ce que je dis comme le dernier mot ». Et c'est vraiment un point de vue de chercheur. Ce qui m'intrigue chez Lacan, que j'ai expliqué un peu, c'est qu'il montre très rarement sa clinique à lui, voilà. Alors que Freud est plus généreux.

Bruno Carignano : - Donc, croyezvous que cela pose un problème au niveau de sa transmission en psychanalyse ?

Paul-Laurent Assoun : - Ça a eu des effets, voilà ! Moi, c'est une question qui m'intéresse. Voyez, si on me demandait ma carte d'identité : " est-ce que finalement vous êtes lacanien ? ". C'est une question que, par définition, je voudrais reposer d'une façon tout à fait différente. Très concrètement dans mon trajet, étant freudien d'emblée, quand j'ai écrit Freud, la philosophie et les philosophes, et dans mes premiers livres, il n'y avait pas un mot sur Lacan. Je l'avais lu, mais je ne le sentais pas. Quand j'ai été invité à faire des cours à Paris 8 - heureusement, c'est un département très vivant lacanien, fondé par Lacan. Eh bien là, j'en ai été de plus en plus intéressé, mais je faisais toujours le passage de l'un à l'autre, voilà. Pour montrer ce que ça devient.

J'ai rencontré Lacan (enfin, c'est l'anecdote). Je ne suivais pas son séminaire, peutêtre parce que, bon, je n'étais pas prêt à suivre un mouvement de foules. Peut-être Freud était un père mort à un moment. J'ai réfléchi là-dessus dans mon analyse. Mais effectivement, voyez, je m'y suis entrainé, pourquoi pas ? Enfin, à un certain moment, je crois que c'était Charles 
Melman à l'époque qui effectivement m'a dit: «Lacan veut vous voir ». Évidemment, ça me flattait beaucoup. Moi, j'étais un jeune agrégé de philo. Je dois avoir eu vingtquatre ans. C'est vrai que j'avais déjà publié ce livre. Et voilà, je suis allé le voir, mais, comment dire ? il ne m'a rien dit. Certains m'avaient dit, ce qui me flattait beaucoup à l'époque, qu'il avait annoté Freud, la philosophie et les philosophes. Lacan était quelqu'un aussi qui voulait tout le temps apprendre - la topologie, etcétéra.

C'est une anecdote personnelle, mais j’y vais, je l'appelle. Il a réagi avec une voix un peu particulière : "Venez la semaine prochaine à la même heure ». Il a fallu que je vois qu'il était 15 heures 12 . Il s'est assis devant moi et il m'a regardé. Alors, à ce moment-là, je lui ai dit — pour meubler la conversation - : « je pense qu'enfin vous avez voulu me voir, quoi. Je crois que vous avez été intéressé par mon travail. Qu'estce que vous pensez de Freud, la philosophie et les philosophes ? "C'était un peu à côté de la plaque. Il me dit à ce moment-là: " qu'est-ce que vous voulez que je vous dise ? C'est excellent ». Alors, en même temps - après j'ai mieux compris " c'est excellent » c'est ce que je voulais qu'il me dise. Mais, en même temps, il devait trouver ça bon parce qu'il l'utilisait. Alors, je lui ai dit : "j’enseigne déjà au Département de Paris 8, chez vous " [rires]. Alors, à ce moment-là, il me dit : " Ah bon, vous n'êtes pas tout à fait un inconnu ». Lacan voulait quand même qu'on se rallie à lui. Mais mon idée, ce n'était pas d'être lacanien, j'avais très peur des institutions qui tournent mal pour les gens qui y sont. Je n'ai jamais connu la haine institutionnelle, mais il faut bien s'identifier à quelque chose, comme dit lui-même. Donc, j'étais prêt à m'identifier, mais enfin, les gens de la SPP [Société Psychanalytique de Paris] avaient rendu hommage à certains de mes travaux, il n'avait aucune raison de ne pas discuter avec eux. Et à ce moment-là, il m'a dit : " bon, je vais vous faire donner un enseignement digne de vous ". C'est très amusant [rires] : " digne de vous ». Parce qu'à l'époque, à Paris 8 , à un Département où il y avait Jacques-Alain Miller, il y avait des enseignements qui étaient recommandé avec une petite étoile, un petit astérisque, et c'étaient les titulaires qui les faisaient. Et donc j'ai eu droit à ce petit astérisque, ce qui faisait qu'on pouvait me considérer comme titulaire. Mais c'était la petite marque, le petit trait unaire [rires]. Voilà, ça a été mon seul rapport avec Lacan.

Il y a des moments où ils me disent : c'est dommage de ne pas faire groupe. Mais, j'avais très, très peur de perdre du temps, ça c'était un point. Il y a des groupes qui apportent beaucoup. Même les cartels, il y en a beaucoup à dire, des choses qui ne vont pas du tout pour moi.

Mais c'est parce que quand même Lacan m'avait donné ce feu vert... Moi, je trouve que cela résume un peu l'autonomie — « l'autonomie, c'est la psychologie du moi ». Ce n'est pas ça. C'est le fait d'effectivement constituer son propre domaine de recherche. Sinon vous devenez la voix de votre maître. Par contre, le maître, il est très, très important, c'est un phénomène de pouvoir. S'il y a une place à prendre, j'apprends Lacan par cœur et, du coup, je deviens la voix de mon maître. Mais ce n'était pas ça Lacan. Et Lacan se méfiait beaucoup de ses disciples. D'ailleurs, il disait : «je vous enseigne, c'est tuant ». Mais, en même temps, il avait besoin de ses disciples, pour le soutenir. 
Bon, tout ça, voyez, j'ai connu tous ces effets, mais sans jamais m'aliéner. C’est ça qui est très précieux, mais pas par souci de l'indépendance, ou de faire tout, tout seul, mais parce qu'il faut pouvoir compter sur ses propres forces. Donc, il me semble, j'ai une chère identification freudienne, c'est ça [rires], mais non, c'est normal. C'est qu'effectivement Freud, lui, est aussi un homme des institutions. Il aimait toutes les relations sociales, il était au centre d'un énorme mouvement social. Le mouvement psychanalytique, c'est un mouvement social qu'il a créé. Freud a créé aussi des gens, des analystes, qui ne sont pas toujours à la hauteur, voyez. Il y a des tas de choses qu'on pourrait dire, mais ce sont des découvreurs. Freud dit : moi, ce que je suis, c'est un découvreur. C'est marrant parce que, du coup, c'est un conquistador. C'est très curieux ce signifiant espagnol chez Freud.

Bruno Carignano : - Donc, une toute petite dernière question pour finir. D'après vous, quelle lecture peut-on faire du mouvement féministe du point de vue de l'anthropologie psychanalytique?

Paul-Laurent Assoun : - Une question délicate pour la fin [rires]. Les femmes ont mené un combat — et là je parle avec le langage des militantes, les suffragettes parce qu'il y avait quelque chose d'une position impossible faite à la femme, une répression socio-politique, c'est vrai. Mais quelque chose qui les mette dans une position impossible que Freud, qui n'était pas du tout féministe, repère. Par exemple, on leur demande, et ça revient avec la question du harcèlement sexuel, on leur demande d'être dans une position : elles n'ont pas le droit d'exercer la pulsion, mais elles sont un objet pulsionnel. Tout ça s'est heureusement déplacé, décalé, etcétéra. Il y a toujours quelque chose de décalé dans ce lien.

Donc, c'est une idéologie le féminisme. On a besoin des idéologies. D'une certaine manière, à chaque fois qu'il y a des idéaux, il y a des idéologies. Il y a une idéologie psychanalytique dans ce sens, dès que je tiens un discours ; la preuve, c'est que c'est souvent malinterprété, etcétéra. Donc, tout discours est du semblant dit Lacan, voilà. Alors, la question - là où ça tourne mal, ça tourne mal, mais c'est inévitable c'est quand on construit une anthropologie imaginaire avec des bons sentiments. Ça, c'est catastrophique, et ça devient une répression — " ah, mais si vous dites ça, c'est parce que vous avez une idéologie ». Rien ne doit nous retenir de dire, de s'autoriser à dire quelque chose, à partir du moment où on croit pouvoir dire que ça vient du réel inconscient.

Alors, il y a une idéologie féministe de ce genre, et aussi pour la question de l'homosexualité, enfin. Comment est-ce qu'un analyste peut avoir une idéologie répressive sur l'homosexualité ? Mais ça ne lui passe pas par la tête normalement dans son acte analytique. Alors, la théorie du genre a été créée et utilisée enfin du même mouvement. C'est une théorie grammaticale - du genre - qui s'est substituée à la logique de la sexuation, voilà. Donc, là il faudrait parler longuement. Effectivement, qu'estce que ce serait un féminisme du réel ? Il y a beaucoup de femmes qui travaillent dans ce sens. Il n'y a pas de guerre des sexes, je précise bien : il y une antinomie de la condition, mais il n'y a pas de guerre des sexes. D'abord, le sexe est quelque chose qui d'emblée est dans le désir. Donc, 
l'homme et la femme ne se battent pas. Ils se battent dans le lit, voyez ce que je veux dire. Il y a là quelque chose qui est très important. Par contre, la contradiction sociale qui travaille le sexuel, la production des discours, c'est foncièrement défavorable à la femme. Alors, je peux dire très rapidement, il nous faut - il y a déjà des femmes de ce genre - un féminisme du réel. Si je commence, dès que je parle : " est-ce que c'est bon pour la cause que je défends ? ", c'est cuit, voilà. Ça, c'est un point qui est absolument essentiel. Mais ce n'est pas un hasard s'il a fallu un mouvement pour faire cesser une partie du scandale de cette répression, voilà.

Le fait est que Freud n'est pas un révolutionnaire, on sait que ce n'est pas un féministe, mais probablement parce qu'il croit à la continuité des structures. Ce qui fait que l'homme et la femme sont égaux dans le fait qu'ils sont tous les deux tarabustés par l'angoisse de castration, et tournent ensemble dans un ballet où chacun essaie de prendre sa position. Par contre, c'est quelqu'un qui, dans sa clinique, montre impitoyablement le sort qui est fait à la femme, et qui met en valeur le vrai désir de la femme. Naturellement, il ne se fait pas un porte-voix féministe, ni même de la jeune homosexuelle, qui, d'ailleurs, se comportait d'une façon insupportable. Elle défendait son désir, mais elle trafiquait les rêves. Sidonie Csillag, comme on l'appelle maintenant, elle se réjouissait, avec sa copine, avec la dame, de dire " tiens, aujourd'hui, à ce crétin on va lui faire des faux rêves ". Mais Freud disait : "c'est bizarre ", parce qu'il sait lire, voilà. Mais à quatre-vingt-dix-neuf ans — on l'a retrouvée juste avant sa mort —, elle traite encore Freud de crétin. Donc, je me pose des questions : est-ce qu'il n'y a pas quel- que chose de la haine du père qui conduit à la haine de la psychanalyse ? Bon, mais tout ça, on peut dire, ce sont des faits historiques, voilà.

Cet entretien a été très intéressant pour moi parce que j'ai eu une occasion de montrer très directement mon trajet, et mon désir.

Bruno Carignano : - Donc je vous remercie beaucoup, ça a été vraiment intéressant du point de vue théorique et, en plus, vous avez rajouté quelques anecdotes personnelles...

\section{Notes}

1 Safouan, Moustapha (1974). Études sur l'Edipe. Paris : Seuil, p.116.

\section{Pr. Paul-Laurent Assoun:}

Professeur émérite de l'Université de Paris (ex-Université Denis Diderot, Paris 7). Psychanalyste praticien adhérent de l'association Espace Analytique. Auteur de nombreux articles et plusieurs ouvrages de psychanalyse.

Mg. Bruno Carignano: Psychanalyste. Doctorant en Cotutelle par convention internationale entre l'Université Paris Diderot -CoMue Sorbonne Paris Cité, Centre de recherches psychanalyse, médecine et société (CRPMS)- et l'Universidad Nacional de Rosario, Facultad de Psicología, Secretaría de Estudios de Posgrados, Doctorado en Psicología. Professeur Titulaire des Chaires Psychanalyse Freud II, Psychanalyse École Française, Atelier du mémoire, Psychopathologie II (UCES, Argentine). Enseignant (Jefe de Trabajos Prácticos) dans la Chaire Psychanalyse II, Universidad Nacional de Rosario (Argentine). 